prophylaxis was extended to neonates of birth weight of 1500 grams and below. The rate of invasive fungaemia was monitored as part of an ongoing COI project to reduce nosocomial infection rates in the NICU.

Results 799 VLBWI were admitted during the study period out of which 227 were ELBWI. There were no differences in birth weight, gestation and gender distribution.

Abstract 1164 Table 1 Episodes \& Rate of Fungaemia

\begin{tabular}{lcccccccc}
\hline & $\mathbf{2 0 0 4}$ & $\mathbf{2 0 0 5}$ & $\mathbf{2 0 0 6}$ & $\mathbf{2 0 0 7}$ & $\mathbf{2 0 0 8}$ & $\mathbf{2 0 0 9}$ & $\mathbf{2 0 1 0}$ & $\mathbf{2 0 1 1}$ \\
\hline Episodes & 13 & 3 & 2 & 1 & 1 & 0 & 2 & 0 \\
Number of Vlbwi & 92 & 91 & 99 & 93 & 100 & 99 & 95 & 97 \\
Rate & $14 \%$ & $3.3 \%$ & $2 \%$ & $1.1 \%$ & $1 \%$ & 0 & $2.1 \%$ & 0 \\
\hline
\end{tabular}

$\mathrm{P}<0.001$.

Conclusions The introduction of a prophylactic oral nystatin administration policy was associated with a significant reduction in invasive fungal infection among high risk neonatal population.

\section{RECTAL SWABS: AN INCREASINGLY IMPORTANT COMPONENT OF NICU INFECTION SURVEILLANCE PROGRAMIMES?}

doi:10.1136/archdischild-2012-302724.1165

'J Suviste, 'J Gray, ${ }^{2}$ Morgan, 'M Patel. 'Department of Microbiology; ${ }^{2} \mathrm{NICU}$, Birmingham Women's NHS Foundation Trust, Birmingham, UK

Background and Aims Gram-negative bacteria present an increasing threat to NICU babies. Because the gastrointestinal tract is the primary colonisation site, we added rectal swabbing to routine admission and weekly screening of babies from September 2010. We consider here the impact of this strategy on clinical and infection control management.

Methods Rectal swabs were cultured for the following Gramnegative bacteria of interest (GNBi): Serratia; gentamicin-resistant \&/or ESBL-producing Enterobacteriaceae; Pseudomonas aerugionsa (PA). Colonised babies were isolated, but were not treated with antibiotics unless clinically indicated.

Results GNBi (except PA) were isolated from 55 (2.6\%) of 2101 admissions, September 2010-March 2012: 21 gentamicin-resistant Enterobacteriaceae; 9 ESBL-producing Enterobacteriaceae; 25 Serratia. 45 of the GNBi were detected in rectal swabs: in $38(64.7 \%)$ rectal swabs were the first, and in $28(33.3 \%)$ the only, culturepositive samples. Only one baby had GNBi infection (bacteraemia on the same day as a positive rectal swab).

There were 13 instances of $2-3$ babies having the same bacterium within 7 days of each other. In one case, seven babies were found with Serratia over 7 days. PA results are not shown for the first 7 months, because of an exceptional event causing PA colonisation then. After April 2011 there were 5 cases (4 detected on rectal swabs: the only positive site in 3 ): there was no clustering of these cases.

Conclusion An unexpectedly high proportion of NICU babies had GNBi. The high frequency of patient-to-patient transmission suggests that rectal screening can be an important tool in controlling these bacteria.

\section{PERFORMANCE OF THE DEFINITIONS OF THE SYSTEMIC INFLAMMATORY RESPONSE SYNDROME AND SEPSIS IN NEWBORNS}

doi:10.1136/archdischild-2012-302724.1166

N Hofer, W Mueller, B Resch. Medical University of Graz, Graz, Austria

Background and Aims In 2002 the International Pediatric Sepsis Consensus Conference created definitions for the systemic inflammatory response syndrome (SIRS) and sepsis adoptable for paediatric patients. We aimed to examine the applicability of the pediatric definitions of SIRS and sepsis to newborns in the diagnosis of early (EOS) and late onset sepsis (LOS).

Methods Retrospective cohort study including

1. all term newborns hospitalized within the first 24 hours of life and

2. all infants with episodes of suspected LOS with a corrected gestational age of $>37$ weeks at onset of LOS from 2004 to 2008.

Results Thirteen of 245 newborns included had culture proven EOS (5\%) and 5 newborns had culture proven LOS. SIRS and sepsis criteria applied to $38 \%$ of EOS positive infants and to $100 \%$ of LOS positive infants. The two major diagnostic criteria white blood cell count and fever/hypothermia, of which at least one has to apply for fulfilling SIRS and sepsis criteria, had a sensitivity of $15 \%$ and $38 \%$ in diagnosis of EOS and of $100 \%$ and $80 \%$ in diagnosis of LOS, respectively.

Conclusions The definitions of SIRS and sepsis applied to all cases of culture proven LOS. However, the single diagnostic criteria were insensitive in diagnosis of culture proven EOS with thus wrong classification of more than $60 \%$ of all cases. An evidence based approach to find the appropriate criteria for defining EOS in newborns is needed.

\section{DOES MATERNAL INTRAPARTUM ANTIBIOTICS PROLONG THE INCUBATION TIME REQUIRED FOR BLOOD CULTURES TO BECOME POSITIVE FOR INFANTS WITH EARLY-ONSET SEPSIS?}

doi:10.1136/archdischild-2012-302724.1167

'S Sarkar, ${ }^{2}$ SS Sarkar, ${ }^{3}$ B Bhagat, 'V Bhatt-Mehta. 'Department of Pediatrics, Division of Neonatal-Perinatal Medicine; ${ }^{2}$ Research Volunteer, Division of Neonatal-Perinatal Medicine, University of Michigan Health System; ${ }^{3}$ Pediatrics, St Joseph Mercy Hospital, Ann Arbor, MI, USA

We hypothesize that maternal intrapartum antibiotic treatment delays the growth of organism in the blood culture obtained during the workup for infants with suspected early-onset sepsis (EOS). The aim is to determine if maternal intrapartum antibiotic treatment prolongs the time to blood culture positivity in infants with EOS.

Methods Single center, retrospective review of infants with blood culture-proven EOS over a 12 years period. EOS was defined by isolation of a pathogen from blood culture drawn within 72 hours of birth and antibiotic treatment for $\geq 5$ days. The automated bacteremia detection was with BacTAlert Peds bottles.

Results Among 88 infants with positive blood culture; 38 were deemed to have EOS, and 50 were deemed contaminants. Seventeen with EOS did not receive intrapartum antibiotics and had blood cultures drawn for being symptomatic after birth. The other 21 infants received intrapartum antibiotics and had EOS workup primarily for maternal chorioamnionitis. The median (IOR) time to blood culture positivity in all 38 infants with EOS was $19.7 \mathrm{~h}(16.5 \mathrm{~h}, 22.5 \mathrm{~h})$, and the organisms grown were: Escherichia Coli in 17, Group B Streptococcus in 10, Alpha hemolytic Streptococci in 6, and other organisms in 5. The median (IOR) incubation time to blood culture positivity was not different in infants who received intrapartum antibiotics compared to infants who did not (19.6 h, IOR $16 \mathrm{~h}-28 \mathrm{~h}$ versus $19.5 \mathrm{~h}$, IOR $17.2 \mathrm{~h}-21.6 \mathrm{~h}, \mathrm{p}=0.7489$ ).

Conclusion Maternal intrapartum antiobiotic treatment did not delay the time to blood culture positivity in infants with EOS NEONATAL INTENSIVE CARE UNIT IN RURAL WESTERN INDIA

doi:10.1136/archdischild-2012-302724.1168 